\title{
Short-time critical dynamics of the Baxter-Wu model
}

\author{
Everaldo Arashiro* and J. R. Drugowich de Felício ${ }^{\dagger}$ \\ Departamento de Física e Matemática, Faculdade de Filosofia, Ciências e Letras de Ribeirão Preto, Universidade de São Paulo, \\ Avenida Bandeirantes, 3900-CEP-14040-901, Ribeirão Preto, São Paulo, Brazil
}

(Received 22 November 2002; published 29 April 2003)

\begin{abstract}
We study the early time behavior of the Baxter-Wu model, an Ising model with three-spin interactions on a triangular lattice. Our estimates for the dynamic exponent $z$ are compatible with results recently obtained for two models which belong to the same universality class of the Baxter-Wu model: the two-dimensional fourstate Potts model and the Ising model with three-spin interactions in one direction. However, our estimates for the dynamic exponent $\theta$ of the Baxter-Wu model are completely different from the values obtained for those models. This discrepancy could be related to the absence of a marginal operator in the Baxter-Wu model.
\end{abstract}

DOI: 10.1103/PhysRevE.67.046123

PACS number(s): 64.60.Ht, 75.10.Hk, 02.70.Uu

\section{INTRODUCTION}

Since the work of Janssen et al. [1] and Huse [2] point out that universality and scaling are present at the early stage of the time evolution of dynamic systems, several statistical models have been investigated using the Monte Carlo simulations in the short-time regime [3]. This kind of investigation avoids the critical slowing down effects and provides an alternate method for calculating the dynamic exponent $z$, the static exponents $\beta$ and $\nu$ as well as the new dynamic exponent $\theta$ that governs the anomalous behavior of the nonequilibrium magnetization when the system is quenched to the critical temperature $T_{c}$. Using this different approach the kinetic Ising and Potts models [4-8] were exaustively studied in order to confirm available results and to enlarge our knowledge of critical phenomena, including the critical relaxation of the early time dynamics.

In this paper, we revisit the Ising model with three-spin interactions on a triangular lattice, called Baxter-Wu (BW) model after its exact solution obtained 30 years ago [9]. The motivation arises from the disagreement between results for the exponent $z$ obtained for the BW model and for two other models - the two-dimensional four-state Potts model and the Ising model with three-spin interactions in one directionwhich are known to belong to the same universality class. The BW model consists of Ising spins $\left(S_{i}= \pm 1\right)$ on a triangular lattice with the Hamiltonian

$$
H=-J \sum_{\langle i, j, k\rangle} S_{i} S_{j} S_{k}
$$

where the sum involves the product of the spins on each triangle of the lattice. As the Ising model on a square lattice this model undergoes a phase transition at the critical temperature $K_{c}=J / k T_{c}=0.5 \ln (1+\sqrt{2})$. The ground state is fourfold degenerate (see Fig. 1) and the relevant symmetry is semiglobal (the Hamiltonian is invariant under reversal of all of the spins belonging to two of three sublatices into which the original lattice can be decomposed). The BW model ex-

\footnotetext{
*Email address: everaldo@dfm.ffclrp.usp.br

†Email address: drugo@usp.br
}

hibits the same static critical exponents $(\alpha=\nu=2 / 3$ and $\eta$ $=1 / 4$ ) $[10]$ as those of the two-dimensional four-state Potts model and of the Ising model with three-spin interactions (IMTSI) in one direction [11]. However, finite-size studies of the BW model have good convergence, without running into the known difficulties encountered in the studies of these other models. Several authors [12] have attributed that difference to a dangerous marginal operator present in the fourstate Potts and IMTSI models which does not have a counterpart in the BW model.

Although the static critical behavior of these systems is well understood, only a few results are known regarding dynamical properties. For instance, much work it is needed to unravel the interesting question of a possible extended universality, i.e., if these three models (BW, four-state Potts, and the IMTSI) exhibit the same exponent $z$. Previous estimates of the critical exponent $z$ for the four-state Potts model are scattered between 2 and 4 [13]. The most recent results, however, suggest values close to $2.3[14,15]$. Short-time simulations for the BW model were first performed by Santos and Figueiredo [16], who found $z=2.07(1)$, a very small value when compared to the values obtained for the same exponents of the four-state Potts and IMTSI models $[14,15]$. Moreover, they stress that this value of $z$ is very close to the dynamic exponent of the the two-dimensional (2D) Ising model when a Glauber dynamics is used [17]. For the new dynamic exponent $\theta$ related to the critical initial slip of the order parameter, situation is even worse. Okano et al. have conjectured [4] that for the four-state Potts model the exponent $\theta$ should be negative and close to zero. This result was only recently confirmed [18]. For the IMTSI model, two independent estimates were obtained $[15,19]$ and fall in the range $-0.04<\theta<0.07$, whereas estimates for the $\mathrm{BW}$ model are not available.

Motivated by our recent results for the IMTSI model [15], we decided to investigate the short-time critical dynamics of the BW model in order to check the value of the dynamic exponent $z$ and the utility of the exponent $\theta$ in detecting the presence of a marginal operator $[5,6,20]$. In the following section we calculate the exponent $\theta$ studying the evolution of the order parameter for different values of the initial sublattice magnetization $m_{0}=(3 / N) \sum_{i} S_{i}(0)$, where the index $i$ runs over only one kind of sites of the triangular lattice 


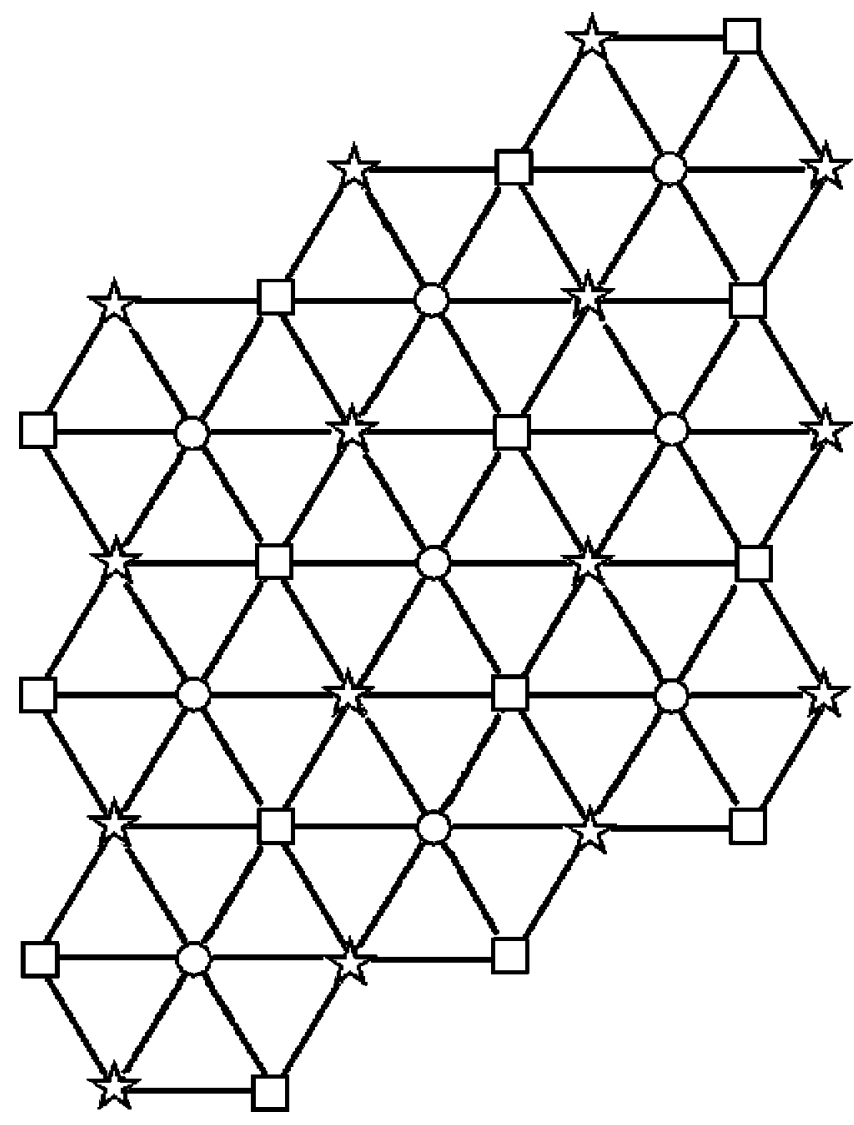

FIG. 1. The Baxter-Wu model is defined on a triangular lattice where the Ising spins $\left(S_{i}= \pm 1\right)$ are coupled by nearest neighbor three-spin interactions. The lattice can be divided into three sublattices, characterized in the figure by circles, squares, and stars. Each symbol represents a class of spins which does not interact with each other. The ground state is fourfold degenerate, corresponding to the three configurations with up spins in two sublattices and down spins in the other, besides the obvious configuration of spins up in all the sublattices.

(circle, square or star points in Fig. 1) and $N$ is the number of spins. In addition, we estimate the same exponent calculating the time correlation of the order parameter [21]. In Sec. III we summarize our results for the dynamic critical exponent $z$. In Sec. IV we list estimates for the correlation length exponent $\nu$ and for the index $\beta$ of the magnetization. Finally, in section $\mathrm{V}$ we present our conclusions.

\section{THE DYNAMIC EXPONENT $\theta$}

Janssen et al. [1] have shown on the basis of the renormalization group theory that, at an early stage of the time evolution, quantities such as the magnetization and its moments follow a power law when the parameters of a dynamical system are adjusted to their critical values. The exponent $\theta$ that characterizes the time evolution of the magnetization is independent of the known set of static critical exponents and even from the dynamic exponent $z$. However, like other exponents $\theta$ seems to depend on general properties such as dimensionality of the spin and either symmetry of the Hamiltonian or the evolution rules. Such universal behavior was

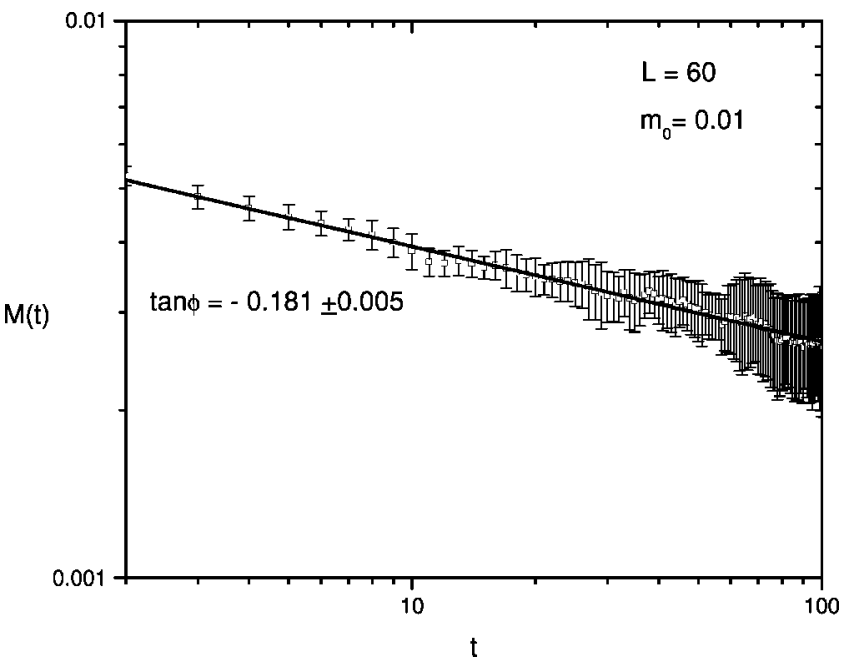

FIG. 2. Power law behavior of the magnetization for samples with initial magnetization $m_{0}=0.01$. The error bars were calculated over 10 sets of 60000 samples.

found in two- and three-dimensional Ising models [4,22], two-dimensional three- and four-state Potts models [14,23] as well as in irreversible models with synchronous and continuous dynamics $[21,24,25]$. In addition, nonuniversal behavior was detected for the exponent $\theta[5,6,26,27]$ although always accompanied by a marginal operator.

In this paper we estimate the dynamic exponent $\theta$ by two different techniques. First we quench the system from a high temperature to $T_{c}$ and follow the nonequilibrium relaxation of various realizations of the process with the same initial sublattice magnetization $m_{0}$. In Fig. 2 we show the behavior of the order parameter when $m_{0}=0.01$. As proposed by Janssen et al. [1] it follows the power law

$$
M(t) \simeq m_{0} t^{\theta}
$$

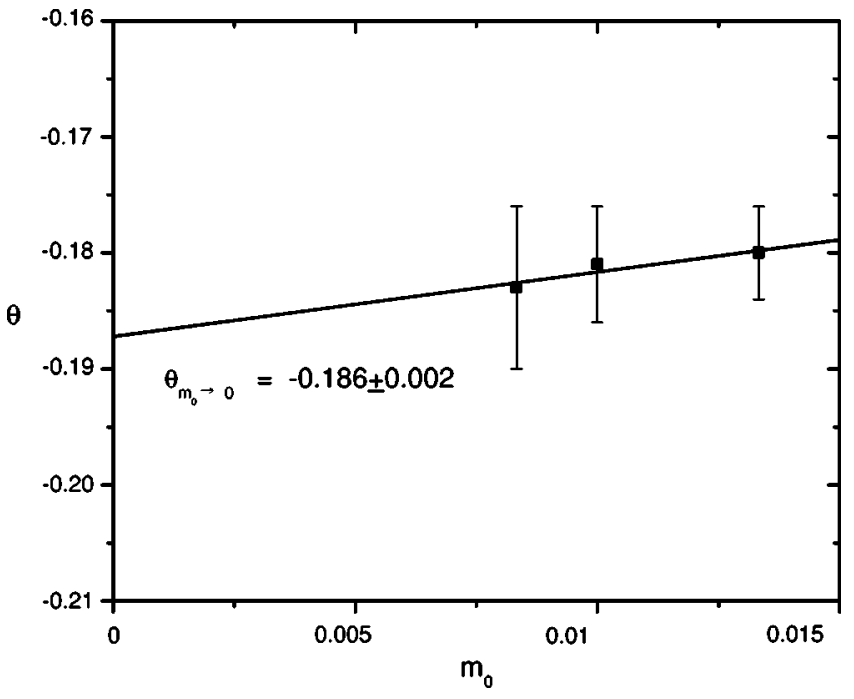

FIG. 3. Dynamic exponent $\theta$ as a function of the initial magnetization $m_{0}$ for the lattice size $L=60$. Each point represents an average over 10 sets of 60000 samples. 


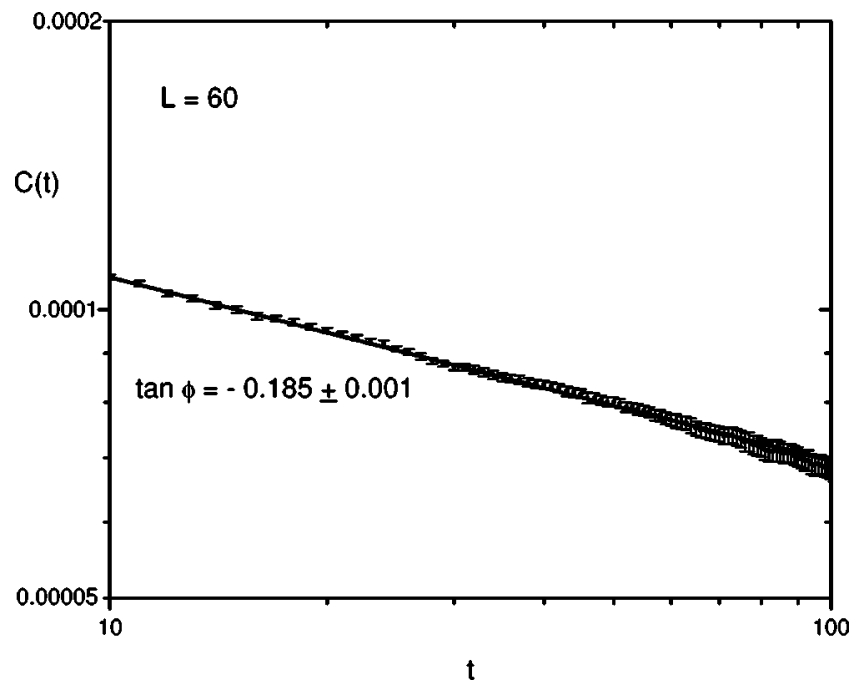

FIG. 4. Time correlation of the total magnetization for samples with $\langle M(t=0)\rangle=0$. Error bars were calculated over 10 sets of 60000 samples.

but here $\theta$ is negative. Figure 3 exhibits the behavior of the exponent $\theta$ for different values of $m_{0}$, as well as a linear fit that leads to the value $\theta=-0.186 \pm 0.002$.

The second technique consists of studying the time correlation of the magnetization

$$
C(t)=\left\langle\sum_{i} S_{i}(t) \sum_{j} S_{j}(0)\right\rangle,
$$

which was shown [21] to behave as $t^{\theta}$ when $\langle M(0)\rangle=0$. In Fig. 4 we show the time dependence of the time correlation $C$. We stress that this approach was shown to be valid when the evolution matrix has up-down symmetry which is not valid in the present case. However, it is possible to show [28] that the $Z(2) \otimes Z(2)$ symmetry of the BW Hamiltonian is sufficient to preserve the above mentioned result. The value of $\theta$ obtained with this technique $(\theta=-0.185 \pm 0.001)$ corroborates the value obtained directly from the behavior of the magnetization. We stress that the time correlation method [21] is numerically more convenient, since we do not need to work with specially prepared initial configurations (with fixed $\left.m_{0}\right)$ nor it is necessary to take the delicate limit $m_{0}$ $\rightarrow 0$.

The results obtained with the two techniques are essentialy the same and fall in the range $-0.188 \leqslant \theta \leqslant-0.184$. They are completely different from the value $\theta=-0.03$ \pm 0.01 encountered for the IMTSI model [15] and from $\theta$ $=-0.047 \pm 0.0033[18]$ obtained recently for the four-state Potts model. Thus, based on our estimates for the exponent $\theta$ of the BW model, we conclude that its dynamic behavior at short time is completely different from that of the IMTSI and four-state Potts models albeit both models have the same static exponents as the BW model. Thus, at least in this case, the exponent $\theta$ seems to be able to capture the subtle difference between models that have the same leading exponents but not the same corrections to scaling.

\section{THE DYNAMIC CRITICAL EXPONENT $z$}

Santos and Figueiredo [16] obtained the exponent $z$ of the $\mathrm{BW}$ model by means of the second-order cumulant $U_{2}$ [8],

$$
U_{2}=\frac{\left\langle M^{2}\right\rangle}{\langle M\rangle^{2}}-1
$$

starting from completely ordered samples $\left(m_{0}=1\right)$. As claimed by Zheng, $U_{2}$ should behave at the critical temperature as a power law

$$
U_{2} \propto t^{d / z}
$$

which would allow us to estimate $z$ without using any other critical exponents. Santos and Figueiredo found $z=2.07(1)$ for $m_{0}=1$ (all sublattices initially with spins up) and 1.96(2) for $m_{0}=-1 / 3$, i.e., spins are initially up in one sublattice and down in the other two. These estimates, obtained with Glauber dynamics [17], are very different from results recently obtained for the four-state Potts model [14] and for the Ising model with three-spin interactions in one direction [15] which both yield values close to 2.3 .

This kind of disagreement has already appeared in the literature when the second cumulant was used to obtain estimates for the exponent $z$ for the three-state Potts model [8] and the IMTSI model [15]. Thus, in order to check if the BW model (which also belongs to the four-state Potts model universality class) exhibits indeed a different value for the dynamic exponent $z$, we have calculated this exponent using three other techniques. To begin with, we estimated $z$ by collapsing the generalized fourth-order Binder cumulant

$$
U_{4}(t, \tau, L)=1-\frac{\left\langle M^{4}\right\rangle}{3\left\langle M^{2}\right\rangle^{2}},
$$

which should only depend on the ratio $t / L^{z}$ when $T=T_{c}$, according to scaling laws valid in the beginning of the evolution. In Fig. 5 we show the Binder cumulant as a function of the time for two different lattices $L=96$ and $L^{\prime}=192$, both starting from ordered states $\left(m_{0}=1\right)$. We also plot in Fig. 5 the cumulant for the larger lattice against the rescaled time $t^{\prime}=2^{z} t$, where $z$ is defined via the relation

$$
U_{4}\left(2^{z} t, 0, L^{\prime}\right)=U_{4}(t, 0, L)
$$

After interpolating the rescaled curve for the lattice $L^{\prime}$, the value of $z$ is selected using the $\chi^{2}$ test [29] yielding the best estimate $2.285(10)$. We applied the same analysis to the cumulant [8]

$$
\widetilde{U}=\frac{\left\langle M^{2}\right\rangle}{|M|^{2}}-1
$$

using $\left(m_{0}=1\right)$ as shown in Fig. 6. The result in this case is $z=2.29(1)$.

Finally, we used mixed initial conditions in order to obtain the function $F_{2}(t)$, given by 


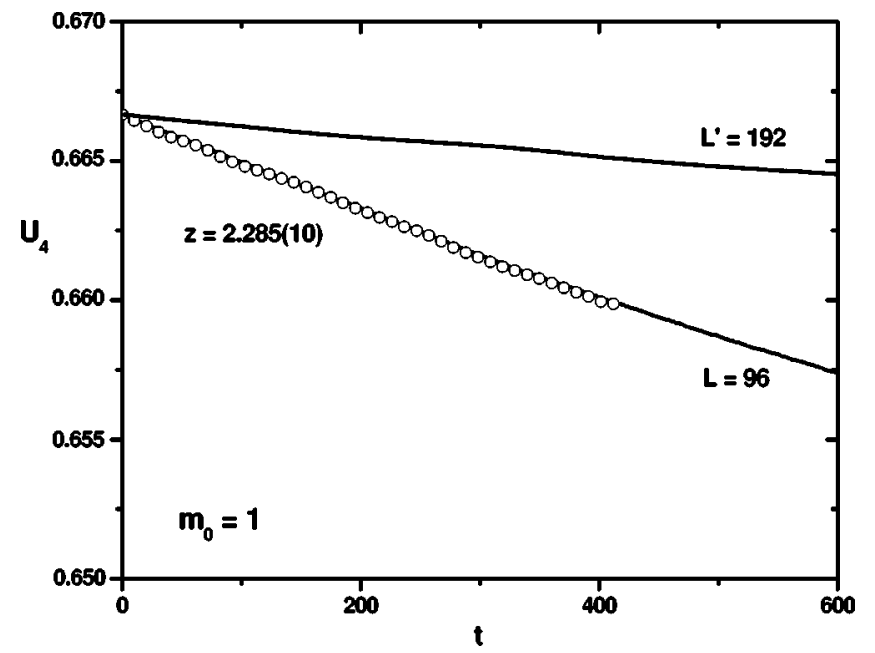

FIG. 5. Binder cumulant $U_{4}(t, 0, L)$ for lattice size $L=96$ and $L^{\prime}=192$ and initial magnetization $m_{0}=1$. The open circles on the continuous line show the cumulant for the bigger lattice rescaled in time with $z$ obtained from Eq. (7).

$$
F_{2}(t)=\frac{\left\langle M^{2}\right\rangle_{m_{0}=0}}{\langle M\rangle_{m_{0}=1}^{2}},
$$

which was shown [14] to behave as $t^{d / z}$. This approach was recently proposed in order to obtain the exponent $z$ with high precision. Actually, curves of $F_{2}$ are straight lines (see Fig. 7) which do not depend on the lattice size (one does not need to rescale the time). Our estimate for $z$ using Eq. (9) and taking values at each 5 steps is $z=2.294(6)$. This value is in complete agreement with the result $z=2.290$ (3) recently obtained [14] for the four-state Potts model and corroborates the above results obtained by different procedures.

It is worth stressing that all the results shown until now were obtained with heat-bath updating, whereas Santos and Figueiredo [16] performed simulations using the Glauber dynamics. In order to rule out any influence of the dynamics on

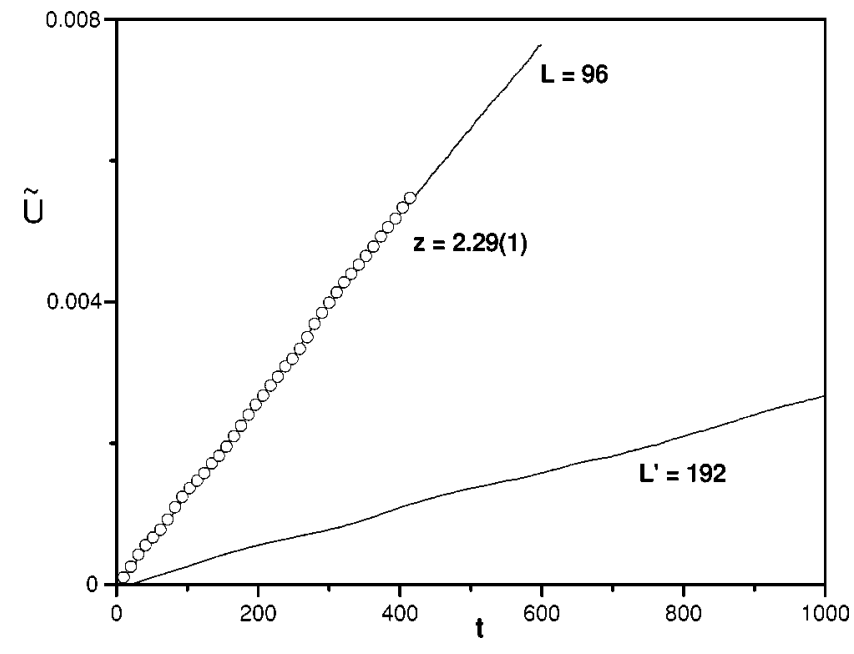

FIG. 6. $\widetilde{U}$ cumulant for $L=96, L^{\prime}=192$ and initial magnetization $m_{0}=1$. The open circles correspond to the rescaled cumulant for the larger lattice with $z=2.29(1)$.

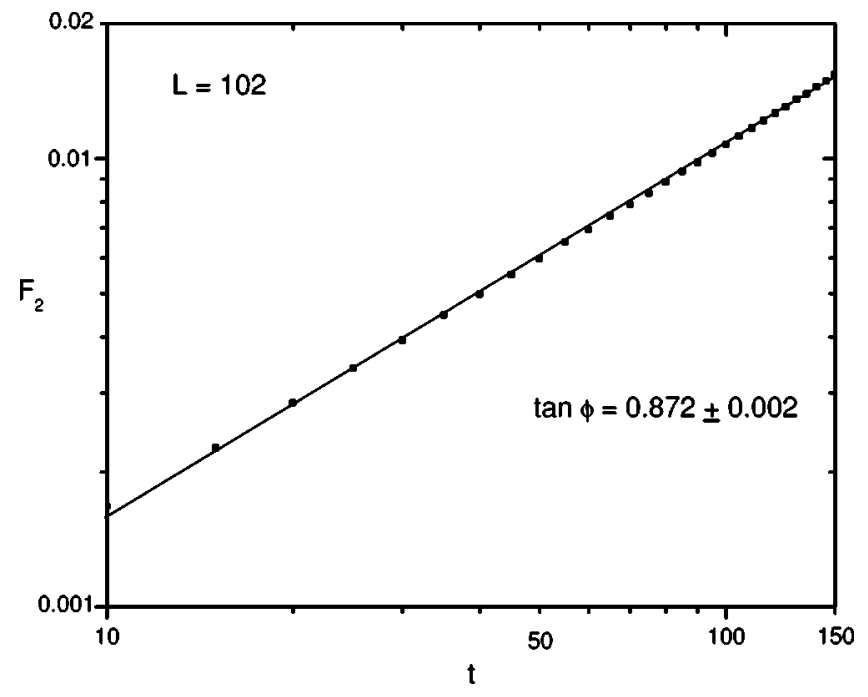

FIG. 7. Time evolution of $F_{2}$ for $L=102$ with mixed initial conditions [Eq. (9)]. The error bars, calculated over 10 sets of 30000 samples, are smaller than the size of the points.

the results we repeated our simulations using the Glauber updating and verified that our result remain essentially the same. In addition, we have used heat-bath updating to calculate the time dependence of the second-order cumulant [Eq. (4)] where the average is always obtained from initially ordered samples $\left(m_{0}=1\right)$. Our best estimate for $z$ is 2.03 \pm 0.01 , which agrees reasonably well with the result by Santos and Figueiredo, indicating that this severe disagreement stems from a difficulty from the scaling for the second cumulant method.

\section{THE THERMAL EXPONENTS $\nu$ AND $\beta$}

Taking the logarithmic derivative of the magnetization [8]

$$
M(t, \tau)=t^{-\beta / \nu z} M\left(1, t^{1 / \nu z} \tau\right)
$$

with respect to $\tau=\left(T-T_{c}\right) / T_{c}$, we obtain

$$
\partial_{\tau} \ln M(t, \tau)=t^{1 / \nu z} \partial_{\tau^{\prime}} \ln \left(\left.F\left(\tau^{\prime}\right)\right|_{\tau^{\prime}=0},\right.
$$

which follows a power law that does not depend on $L$, where $F\left(\tau^{\prime}\right)$ is a scaling function. Thus, a log-log plot of that derivative versus time gives $1 / \nu z$ (see Fig. 8). Once we have obtained the dynamical exponent $z$, the thermal exponent $\nu$ may be determined. Our estimate for the exponent $\nu$ in the short-time analysis is $0.65 \pm 0.01$ which should be compared to the exact value $2 / 3$ from Ref. [30].

Next, we follow the decay of the sublattice magnetization in initially ordered samples $\left(m_{0}=1\right)$. The scaling law

$$
M \propto t^{-\beta / \nu z}
$$

allows us to obtain $\beta / \nu z$ which in turn leads to the exponent $\beta$ by using the previous result for the product $\nu z$. We show a $\log$ - $\log$ plot of $M$ versus $t$ in Fig. 9. A linear fit gives the value $0.0527 \pm 0.0005$ for the ratio $\beta / \nu z$ leading to the estimate 


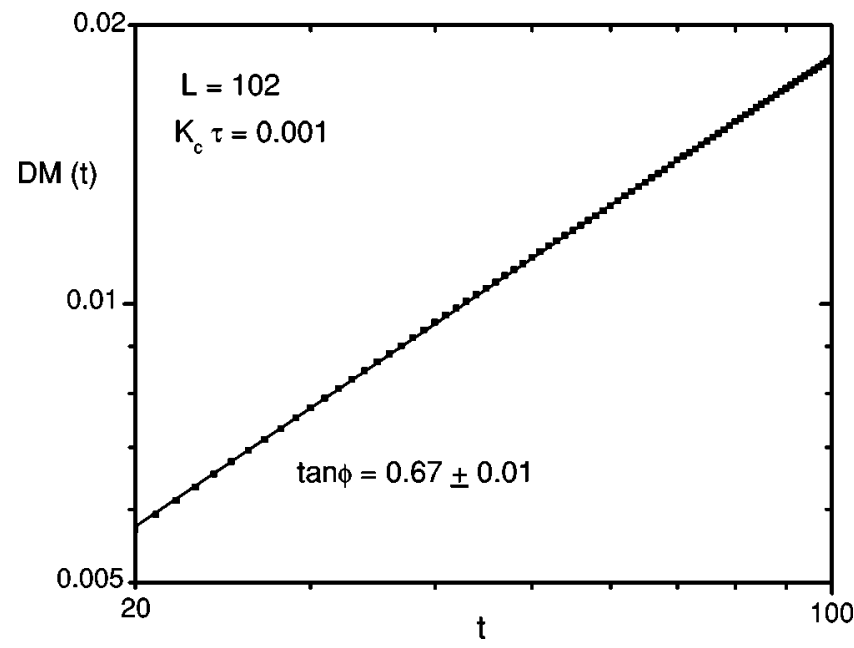

FIG. 8. Logarithmic derivative of the magnetization with respect to the reduced temperature $D M(t)$ as a function of time. Data were obtained for the lattice size $L=102$ using temperature variation $K_{c} \tau=0.001$. The slope corresponds to the ratio $1 / \nu z$, after Eq. (11). The error bars, calculated over 10 sets of 30000 samples, are smaller than the size of the points.

$$
\beta=0.079 \pm 0.002 \text {. }
$$

We stress that our result for $\beta$ is in fair agreement with the exact result 1/12 [9].

Before closing this section we present another estimate for the exponents $\beta$ and $\nu$ obtained from scaling of the socalled Baxter order parameter $M_{B}$ given by

$$
M_{B}=\sum_{\langle i j k\rangle} S_{i} S_{j} S_{k}
$$

where the sum is done over all triangles of the lattice and the indices $i, j$, and $k$ denote sites composing an elementary triangle (plaquette). Barber and Baxter [31] have shown that $M_{B}$ is zero for temperatures below $T_{c}$ and different from

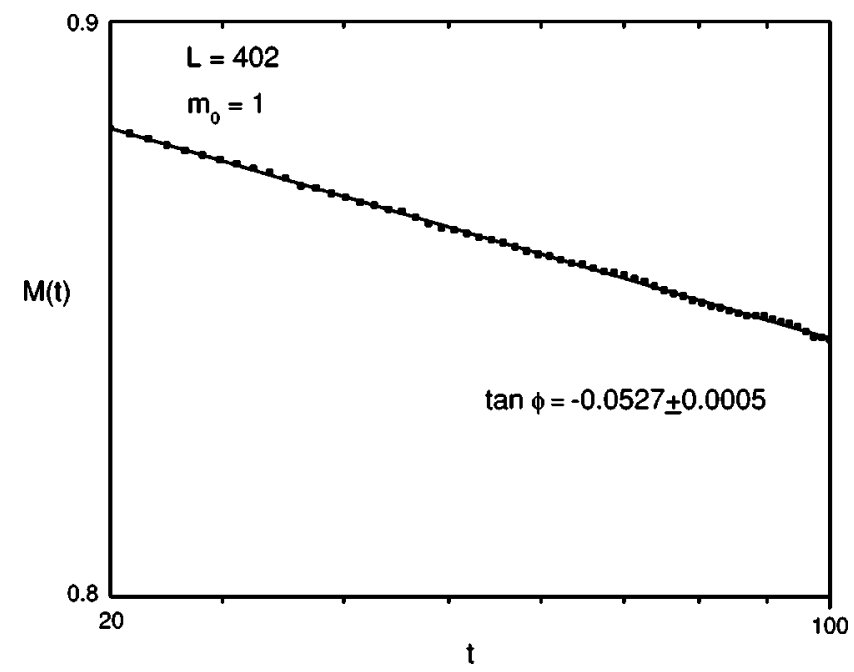

FIG. 9. Log-log plot of the time evolution of the magnetization for initially ordered samples $\left(m_{0}=1\right)$. The error bars (calculated over 5 sets of 1000 samples) are smaller than the size of the points.

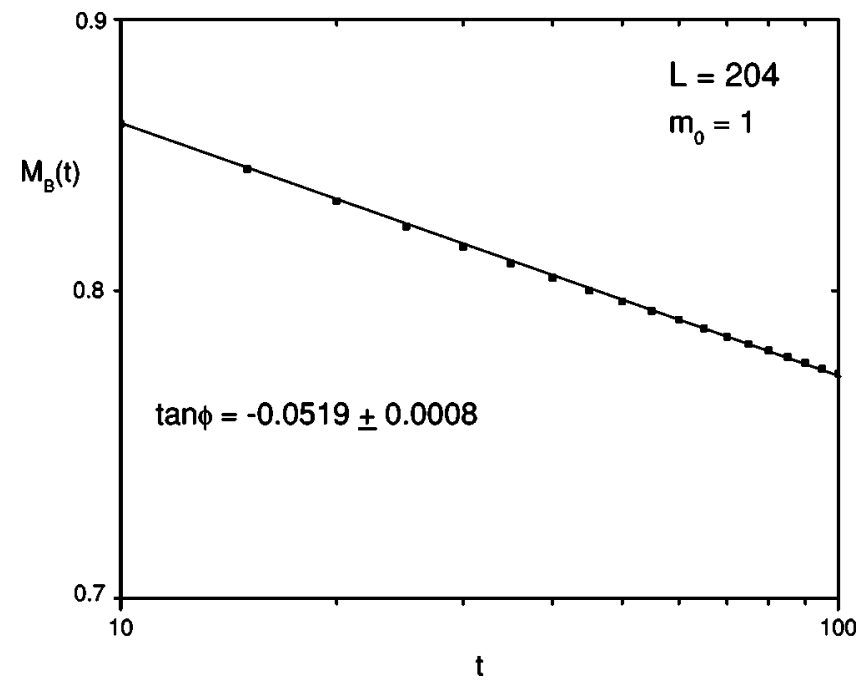

FIG. 10. Time decay of Baxter's order parameter $M_{B}$. Error bars were calculated over 5 sets of 10000 samples.

zero above $T_{c}$. In this sense $M_{B}$ functions as a disorder parameter [32] which goes to zero when $T \rightarrow T_{c}$ from above. However, this new quantity has the same critical exponent as the sublattice magnetization. Thus, the exponent $\beta$ can be obtained following the evolution of Baxter's order parameter. The advantage is that we do not need to divide the system into sublattices. In Fig. 10 we show the decay of the Baxter order parameter as well as the value of the ratio $\beta / \nu z$. In order to eliminate the product $\nu z$ from our result we start again from ordered samples $\left(M_{B}=1\right)$ and use Eq. (11) replacing $M$ by $M_{B}$ to obtain $1 / \nu z$. Figure 11 shows the evolution of the derivative. Our new estimate for $1 / \nu z$ is 0.651 \pm 0.006 , yelding for $\nu$ the value $0.67 \pm 0.01$ in very good agreement with the exact result $2 / 3$ [9]. The new estimate for $\beta$ is $0.080 \pm 0.002$ which is in fair agreement with our previous result and should be compared to the pertinent result $1 / 12$.

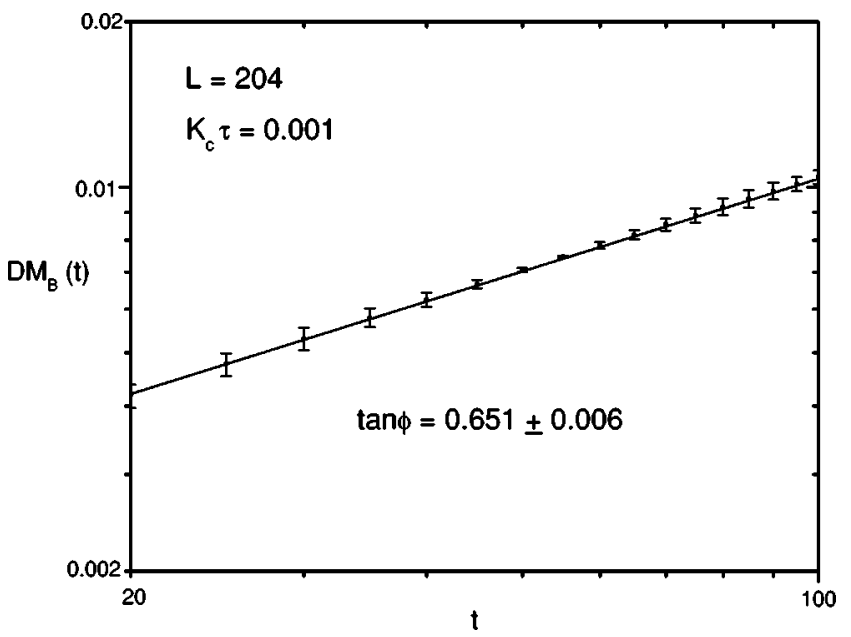

FIG. 11. Logarithmic derivative of Baxter's order parameter $M_{B}$. Data were obtained for the lattice size $L=204$ using temperature variation $K_{c} \tau=0.001$. Five sets of 10000 initially ordered samples $\left(m_{0}=1\right)$ were used in order to obtain the error bars. 


\section{CONCLUSIONS}

We revisited the Baxter-Wu model using the short-time Monte Carlo simulations. Our best estimate for the dynamic critical exponent is $z=2.294 \pm 0.006$. It was obtained by a recently proposed technique [14] that combines simulations performed with different initial conditions. This result is compatible with recently obtained estimates for $z$ in the case of the two-dimensional four-state Potts model [14] as well as for the two-dimensional Ising model with three-spin interactions along one direction [15]. The result for $z$ was confirmed by two other techniques based on the collapse of cumulants. In addition, using scaling relations for the order parameter and its derivative with respect to the reduced temperature $\tau$, we were also able to estimate the static critical exponents $\beta$ and $\nu$. We have also obtained the exponents $\beta$ and $\nu$ using Baxter's order parameter. The results for these exponents are in good agreement with the exact values [9]. However, our estimates for the new dynamic exponent $\theta$ of the Baxter-Wu model are completely different from the values found for that index when studying the two-dimensional Ising model with three-spin interactions in one direction and the four-state Potts model. In this sense, the exponent $\theta$ seems to be able to capture subtle difference between models which exhibit the same leading critical exponents but different corrections to scaling.

\section{ACKNOWLEDGMENTS}

We would like to thank T. Tome for discussions about her result concerning the validity of the correlation method for the BW model prior to its publication. We would also like to thank R. Dickman, N. Caticha, N. Alves, U. H. E. Hansmann, and N. Alves, Jr. for discussions and a careful reading of the manuscript. This work was supported by the Brazilian agencies FAPESP, CAPES and CNPq.
[1] H.K. Janssen, B. Schaub, and B. Schmittmann, Z. Phys. B: Condens. Matter 73, 539 (1989).

[2] D.A. Huse, Phys. Rev. B 40, 304 (1989).

[3] Z.B. Li, L. Schülke, and B. Zheng, Phys. Rev. Lett. 74, 3396 (1994).

[4] K. Okano, L. Schülke, K. Yamagashi, and B. Zheng, Nucl. Phys. B 485, 727 (1997).

[5] C.S. Simões and J.R. Drugowich de Felício, J. Phys. A 31, 7265 (1998).

[6] A.J. Ye, Z.G. Pan, Y. Chen, and Z.B. Li, Commun. Theor. Phys. 33, 205 (2000); N. Alves, Jr. and J.R. Drugowich de Felício, Mod. Phys. Lett. B (to be published).

[7] R. da Silva, N.A. Alves, and J.R. Drugowich de Felício, Phys. Rev. E 66, 026130 (2002).

[8] B. Zheng, Int. J. Mod. Phys. B 12, 1419 (1998).

[9] R.J. Baxter and F.Y. Wu, Phys. Rev. Lett. 31, 1294 (1973).

[10] F.Y. Wu, Rev. Mod. Phys. 54, 235 (1982).

[11] J.M. Debierre and L. Turban, J. Phys. A 16, 3571 (1983).

[12] F.C. Alcaraz and J.C. Xavier, J. Phys. A 30, L203 (1997); 32, 2041 (1999).

[13] L. Arcangelis and N. Jan, J. Phys. A 19, L1179 (1986).

[14] R. da Silva, N.A. Alves, and J.R. Drugowich de Felício, Phys. Lett. A 298, 325 (2002).

[15] C.S. Simões and J.R. Drugowich de Felício, Mod. Phys. Lett. B 15, 487 (2001).
[16] M. Santos and W. Figueiredo, Phys. Rev. E 63, 042101 (2001).

[17] R.J. Glauber, J. Math. Phys. 4, 294 (1963).

[18] R. da Silva and J.R. Drugowich de Felício (unpublished).

[19] L. Wang, J.B. Zhang, H.p. Ying, and D.R. Ji, Mod. Phys. Lett. B 13, 1011 (1999).

[20] K. Oerding and H.K. Janssen, J. Phys. A 26, 3369 (1993).

[21] T. Tomé and M.J. de Oliveira, Phys. Rev. E 58, 4242 (1998).

[22] M. Silvério Soares, J.K. da Silva, and F.C. SaBarreto, Phys. Rev. B 55, 1021 (1997).

[23] L. Schulke and B. Zheng, Phys. Lett. A 204, 295 (1995).

[24] J.F.F. Mendes and M.A. Santos, Phys. Rev. E 57, 108 (1998).

[25] T. Tomé and J.R. Drugowich de Felício, Mod. Phys. Lett. B 12, 873 (1998); A. Brunstein and T. Tomé, ibid. 13, 471 (1999).

[26] Z.B. Li, X.W. Liu, L. Schulke, and B. Zheng, Physica A 245, 485 (1997)

[27] T. Tome, C.S. Simões, and J.R. Drugowich de Felício, Mod. Phys. Lett. B 15, 1141 (2001).

[28] T. Tome, e-print cond-mat/0304218.

[29] W. Press et al., Numerical Recipes (Cambridge University Press, London, 1986).

[30] R.J. Baxter, Exactly Solved Models in Statistical Mechanics (Academic Press, London, 1982).

[31] M.N. Barber and R.J. Baxter, J. Phys. C 6, 2913 (1973).

[32] L.P. Kadanoff and H. Ceva, Phys. Rev. B 3, 3918 (1971). 